\title{
Implicit attitudes towards children with autism versus normally developing children as predictors of professional burnout and psychopathology
}

\author{
Amanda Kelly ${ }^{\mathrm{a}, *}$, Dermot Barnes-Holmes ${ }^{\mathrm{b}}$ \\ ${ }^{a}$ Department of Psychology, National University of Ireland, Maynooth, Co. Kildare, Ireland \\ ${ }^{\mathrm{b}}$ National University of Ireland, Maynooth, Ireland
}

\section{A R T I C L E I N F O}

Article history:

Received 13 April 2012

Received in revised form 17 July 2012

Accepted 17 July 2012

Available online 30 August 2012

\section{Keywords:}

Implicit

Autism

Attitudes

Burnout

Psychopathology

\begin{abstract}
A B S T R A C T
Tutors trained in applied behaviour analysis $(n=16)$ and mainstream school teachers $(n=16)$ were exposed to an Implicit Relational Assessment Procedure (IRAP) designed to assess implicit attitudes towards individuals with autism versus normally developing individuals. Participants also completed a range of explicit measures, including measures of professional burnout and psychopathology. All participants produced more negative biases towards children with autism compared to children who were normally developing. Increased negativity towards autism on the IRAP predicted similar attitudes on some of the explicit measures and also correlated with increased levels of self-reported psychopathology and professional burnout for the tutors working with children with autism. Results suggest that implicit measures of attitudes may provide a marker for professional burnout.
\end{abstract}

(c) 2012 Elsevier Ltd. All rights reserved.

\section{Introduction}

In psychology there has been a long history of interest in people's attitudes, and attempts to measure these attitudes are both varied and ongoing (e.g., Akrami, Ekehammar, Claesson, \& Sonnander, 2006; Amodio, Harmon-Jones, \& Devine, 2003; De Houwer, Crombez, Baeyens, \& Hermans, 2001). It has long been recognised that attitudes can differ and vary in strength, and attempts have been made to quantify and assess the centrality or importance of attitudes for individuals (Fazio, Sanbonmatsu, Powell, \& Kardes, 1986). Some early research on attitudes and their effect on behaviour focused on attitudes toward people with developmental or intellectual disabilities (e.g., Leyser, Kapperman, \& Keller, 1994; Pittock \& Potts, 1988). The importance and need for useful and accurate measurement of professionals' attitudes specifically toward people with disabilities continues to be acknowledged (e.g., Pruett \& Chan, 2006).

Wright (1988) described the unspoken pervasiveness of the bias against people with disabilities in society and related how this can be manifested in rehabilitation practice, education, and research. Attitudes of practitioners can affect people with disabilities in that they frequently have control over rehabilitation monies, services, and information. Therefore if negative attitudes prevail services can be compromised (Pruett \& Chan, 2006). Negative attitudes have also been found to affect working alliances and inhibit the equal status of the client, which in turn can result in further disempowerment of the client (Reeve, 2000). Overall, negative attitudes of rehabilitation professionals toward clients have been shown to translate into less than optimal outcomes (Kaplan, 1982). The importance of accurate measurement of professionals' attitudes

\footnotetext{
* Corresponding author. Tel.: +353868475390.

E-mail addresses: AMANDA.KELLY.2009@nuim.ie, mandiekelly@gmail.com (A. Kelly), Dermot.Barnes-Holmes@nuim.ie (D. Barnes-Holmes).
} 
towards clients be gleaned from the fact that with insight into underlying biases, necessary interventions to improve rehabilitation processes and outcomes could be implemented (Pruett \& Chan, 2006).

The importance of practitioners' awareness of their own potentially negative attitudes toward people with disabilities has also been acknowledged (Pruett \& Chan, 2006). Although the vast majority of rehabilitation professionals are aware that they should have egalitarian attitudes and behaviours toward their clients, some practitioners may endure personal struggles in that they are outwardly expressing attitudes in line with what is thought to be socially appropriate, while attempting to overcome their own biases and assumptions. Recent studies suggest that caring practitioners may suppress or avoid negative thoughts and feelings pertaining to a client population, which in turn produces various forms of psychological stress (Hayes et al., 2004). This may in turn lead to burnout (Barnes-Holmes et al., 2006). Burnout is thought to be a syndrome which fosters feelings of emotional exhaustion, depersonalisation, and reduced personal accomplishment (Maslach, Jackson, \& Leiter, 1996). The consequences of burnout are thought to be potentially harmful for workers, clients, and institutions. Various studies on burnout (e.g., Jackson \& Maslach, 1982; Maslach \& Jackson, 1985; Maslach \& Pines, 1977; Maslach, 1982) suggested that it may lead to deterioration in the quality of care or service provided by professionals in both human services and educational institutions. For both practitioners and teachers burnout was found to be a factor in absenteeism, low morale, personal dysfunction, and various personal problems ranging from increased drugs and alcohol use to marital problems (Lowenstein, 1991; Maslach, Jackson, \& Leiter, 1996). The impact of burnout on service providers' mood and social behaviour may have direct implications for their capacity to maintain a good working environment due to factors such as increased exhaustion and cynicism (Hakanen, Bakker, \& Schaufeli, 2006). Various studies have found that 'mindfulness'; meaning becoming aware of one's own thoughts and feelings, is a useful approach for increasing awareness and responding skilfully to mental processes that contribute to emotional distress and maladaptive behaviour (see Bishop et al., 2004). Thus, an awareness and acceptance of professionals' own negative attitudes toward clients or students may serve to decrease psychological stress and burnout.

Increased knowledge regarding attitudes has been identified as an important factor in enhancing the integration of people with disabilities into society (Wong, Chan, Cardoso, Lam, \& Miller, 2004). For example, Praisner (2003) noted that integration has become a critical part of efforts to improve the delivery of education services to students with disabilities, with focus centred on the placement of these individuals in general education settings. Wilson (2005) acknowledged that in order for inclusion to be successful peoples' attitudes must be addressed. Praisner (2003) found that success of integrating students with disabilities into education settings depended heavily on the willingness of administrators to make decisions that provided appropriate opportunities for these students to remain in general education settings. Individuals in leadership positions are particularly influential. For example, a school principles' attitude may result either in increased opportunities for students' general education, or in limited efforts to reduce the segregated nature of special education services (Praisner, 2003). In fact, positive attitudes of all participants in inclusive settings (i.e., teachers, practitioners, students, parents, administrators, support staff, carers, etc.) are thought to be of the utmost importance, as without it restricted or minimal learning and growth is likely (Kolar \& Dickson, 2002). With such influential effects, the accurate evaluation of attitudes and the subsequent fostering of positive attitude development may be fundamental when considering integration of individuals with developmental and intellectual disabilities.

Considering the impact negative attitudes can have in applied settings, the importance of effective attitude measurement is clear. The literature on the measurement of attitudes is indeed vast (e.g., Wittenbrink \& Schwarz, 2007), but in broad terms there is general agreement that measures should be divided into two types - measures that seek to tap into explicit attitudes and those that seek to tap into implicit attitudes.

Within social psychology it is theorised that explicit attitudes involve controlled processes and require active attention on the part of the individual. For example, upon becoming aware of a situational cue implying the importance of considering ones attitude toward an object, an individual may attempt to retrieve a previously stored evaluation of the attitude object, or actively construct an attitude, both of which are reflective and active processes (Fazio et al., 1986). Several theories thus suggest that explicit measurement of attitudes is particularly valuable for the prediction of deliberate controlled behaviour (see Friese, Hofmann, \& Wanke, 2008). Explicit self-report measures serve to assess such deliberate evaluations.

In contrast, implicitly measured attitudes are assumed to be more important for the prediction of less controlled, more spontaneous behaviour. Petty, Haugtvedt, and Smith (1995) reported that the stronger an attitude is the more likely it is to predict behaviour, and the strength of an attitude was thought to be related to the extent that it is automatic (Fazio et al., 1986). Fazio et al. (1986) argued that in order for an attitude to occur automatically without consideration or reflection, a previously well learned set of associations or responses must exist (e.g., Shiffrin \& Schneider, 1977). Fazio and colleagues also noted that if an attitude is activated automatically upon encountering the attitude object (i.e., social issues, categories of people, specific individuals, or physical objects) it is far more likely to guide the individual's behaviour towards the object than if it is not.

Due to theories that impulsive or automatic processes are important in guiding certain types of behaviour and a belief that implicit cognitions are strongly related to attitudes, stereotypes, and self esteem (Greenwald \& Banaji, 1995), explicit measurement of attitudes has been somewhat overshadowed by attempts to measure implicit attitudes effectively. There are also issues such as response bias effects and socially desirable responding to consider when using measures designed to target explicit attitudes. That is, explicit attitude scales and survey questions will almost always be influenced to some extent by the particular person asking the question or by the whole verbal culture in which the survey is conducted (Silver, Abramson, \& Anderson, 1986). 
In line with the research concerning the automaticity of negative attitudes or prejudices (see Pruett \& Chan, 2006) and its relationship to behaviour, Akrami et al. (2006) acknowledged that any future research on attitudes towards individuals with developmental or intellectual disabilities should focus on "examining these attitudes' more implicit or automatic function using an experimental design analyzing implicit rather than explicit attitudes" (p. 615). They suggested that the assessment of attitudes using implicit measures was an important step in understanding what impact the label or social category of people with disabilities has on our implicit attitudes, and furthermore, on our behaviour and social interactions. Problems such as response bias effects inherent in explicit and self report measures also mean that accurate measurement of explicit attitudes is very difficult. Research on implicit attitude assessment has thus seen researchers attempt to alter performances on implicit measures. If performances on implicit measures are susceptible to manipulation the measure may be of limited utility, in the same way that explicit attitude measurement is of limited utility due to response bias and social desirability. For example, previous studies on the Implicit Association Test (IAT; Greenwald, McGhee, \& Schwartz, 1998) indicated that participants were able to control their responding, particularly when they had some prior experience with the procedure, or were instructed in how to fake their responses (Fiedler \& Bluemke, 2005; Kim, 2003). Another implicit measure however, the Implicit Relational Assessment Procedure (IRAP; Barnes-Holmes, Barnes-Holmes, Stewart, \& Boles, 2010), has been more successful in that participants were found to be unable to fake the IRAP effect (McKenna, Barnes-Holmes, Barnes-Holmes, \& Stewart, 2007). Indeed, even when participants were provided with explicit instructions on how to fake the IRAP the effects are neither removed nor reversed.

Although it has been previously acknowledged that clinicians, educators, researchers, and students may possess negative attitudes towards people with disabilities, there has been limited research designed to measure implicit attitudes toward the disabled (Pruett $\&$ Chan, 2006). A small number of studies have attempted to measure implicit attitudes to a range of disabilities (Thomas, Doyle, \& Vaughan, 2007; Vaughan, Thomas, \& Doyle, 2011; White, Jackson, \& Gordon, 2006), including attitudes to individuals diagnosed with autism (Barnes-Holmes et al., 2006). In the study reported by Barnes-Holmes et al. (2006) professionals with varying levels of experience with individuals with autism were exposed to the Implicit Relational Assessment Procedure (IRAP), which was designed to measure the professional's implicit attitudes to this particular disability. Participants were presented with attribute words that were deemed stereotypically consistent with autism (e.g., sad, bad, difficult, etc.), and attributes that were deemed stereotypically consistent with Normally Developing (e.g., good, easy, calm, etc.) in the presence of the words similar and opposite. It was thought that participants would produce negative biases by responding more quickly to Autism-Negative-Similar than Autism-Negative-Opposite. It was also speculated that the professionals with experience working with individuals with autism would express more positive attitudes to the disorder on explicit measures than professionals with no experience of autism. However it was thought that this difference may be absent on the IRAP.

As predicted, the results of the study indicated that participants with and without experience of autism produced implicit biases on the IRAP that indicated greater positivity towards normally developing children relative to children with autism, with no significant differences between the groups. Responses to questionnaires (i.e., explicit attitudes) however, indicated that the experience with autism group had significantly more positive attitudes to autism than the no-experience group. Research has also shown that participants are unable to fake the IRAP effect (McKenna et al., 2007) rendering it a very useful tool to reliably assess implicit attitudes.

Although Barnes-Holmes et al. (2006) concluded that there was a need to assess stigmatisation and burnout among people working with individuals with autism, the study was preliminary and thus did not attempt to address this issue. In order to pursue this line of research the current study sought to explore whether negative implicit attitudes towards autism could predict burnout and psychopathology among tutors who teach children with autism, and teachers employed in mainstream settings.

This study was deemed to be quite exploratory in nature. However, given previous findings on the relationship between burnout and negative attitudes towards relevant client groups (see Maslach, Jackson, \& Leiter, 1996), it was predicted that implicit attitudes towards children with autism may predict higher levels of self-reported explicit measures of burnout and psychopathology, at least for those teachers who worked with this particular group.

\section{Methods}

\subsection{Participants}

Thirty-two participants in total completed the study. The first group of participants consisted of 16 Applied Behaviour Analysis (ABA) tutors working in a school specifically for children with autism (ABACAS; Applied Behaviour Analysis Centre for Autism Schooling). All 16 ABA tutors were female, and their ages ranged from 22 to 35 . All of the tutors worked exclusively with children with autism on a daily basis in their working week. Students' ages ranged from 6 to 14 years, and diagnoses in the school ranged from moderate to severe autism. The majority of the students were either non-verbal (60\%) or had limited verbal ability in the form of echoed and/or non-functional speech (25\%). Eighty-five percent of the students displayed behavioural issues such as self-injury (defined as any action that the child performs that results in physical injury to the child's own body), physical aggression towards staff, and physical aggression towards other students (physical aggression is defined as any action that the child performs that results in any physical injury to another person, and also includes spitting intentionally at another person). 
The second group of participants consisted of 16 primary school teachers, all of whom worked with normally developing children on a daily basis in their working week. Fourteen of the teachers were female and 2 were male. Their ages ranged from 24 to 40 . The students' ages ranged from 5 to 12 years. None of the teachers had any prior experience teaching children with autism. None of the 32 participants had any previous or direct experience with the IRAP. All individuals presented with normal or corrected-to-normal vision and were native English speaking. All individuals successfully completed the IRAP task and no financial rewards of any kind were offered for participation.

\subsection{Apparatus and materials}

\subsubsection{Implicit attitude measure}

The IRAP was presented within a single computer program, which presented stimuli and recorded all responses. The stimuli employed in the current study consisted of twelve words - six of which (e.g., HAPPY and EASY) were deemed to be positive and consistent with Normally Developing, and six of which (e.g., SAD and DIFFICULT) were deemed to be negative and consistent with Autistic Spectrum Disorder (see Table 1). The response options, "Similar" and "Opposite", were also presented on each trial. Each of the words selected were adopted from previous research by Barnes-Holmes et al. (2006).

\subsubsection{Explicit attitude measures}

Participants were required to complete several explicit measures. The first measure was a 7-point Likert scale. This was designed to assess explicit attitudes towards individuals with autism versus normally developing individuals. The Likert scale was deemed appropriate as an initial measure in that it is acknowledged as a commonly used tool in research to measure attitude; and provides a range of responses to given questions or statements (Cohen, Manion, \& Morrison, 2000). Each Likert contained one of the words that appeared during the IRAP trials. Participants were asked to "indicate the extent to which you find children with autism to be as described in the scale below by circling the appropriate number" or "indicate the extent to which you find normally developing children to be as described in the scale below by circling the appropriate number." Participants were required to circle a number on the scale, each of which had a negative word located at -3 (see Table 1, target deemed consistent with label 1 ) and a positive word located at +3 (see Table 1 , targets deemed consistent with label 2). The number 0 on the scale indicated a neutral response. Positive scores indicated a positive attitude and negative scores a negative attitude.

The second explicit measure was an Attitudes to Autism scale, which was previously designed and used by BarnesHolmes et al. (2006). The scale consisted of statements that related to children with autism or normally developing children; participants are required to enter a number from 1 to 5 (from strongly agree to strongly disagree) beside each statement in order to indicate the level to which they agreed or disagreed with the statement. Responses to the questions were coded such that a mean score above 3 indicated greater positivity towards normally developing children than those with a diagnosis of autism, whereas scores below 3 indicated the opposite (i.e., greater positivity towards autism).

A feeling thermometer was also employed. This is a commonly used measure to determine and compare respondents' feelings about a given person, group, or issue (e.g., Greenwald et al., 1998). It consisted of a printed picture of a thermometer with degrees from $0^{\circ}$ to $100^{\circ}$, with warm/favourable marked at $100^{\circ}$ and cold/unfavourable marked at $0^{\circ}$. Two lined spaces appeared underneath the thermometer in which participants were asked to write down the degrees of warmth versus coldness they felt towards children with autism and normally developing children.

\subsubsection{Explicit non-attitude measures}

The fourth explicit measure was the Depression Anxiety Stress Scale (DASS; Lovibond \& Lovibond, 1995), which consists of 42 statements relating to the extent to which a person may be anxious, depressed or stressed across the past week. Each participant was required to read each statement (e.g., "I found myself getting upset by quite trivial things") and circle a number $0,1,2$ or 3 (ranging from "did not apply at all” to "applied very much/most of the time"). The DASS has been reported

Table 1

The word sets selected for the IRAP.

\begin{tabular}{lcc}
\hline Label 1 & Label 2 & Normally developing \\
\hline Autistic spectrum disorder & Response Option 2 \\
\hline Response Option 1 & Opposite & Targets deemed consistent with Label 2 \\
\hline Similar & Happy \\
\hline Targets deemed consistent with Label 1 & Good \\
\hline Sad & Easy \\
Bad & Calm \\
Difficult & Positive \\
Angry & Pleasant \\
Negative & & \\
\hline
\end{tabular}


to possess good reliability, with the depression, anxiety and stress scales scoring .91 , .84 and .90 , respectively using Cronbach's alpha (Lovibond \& Lovibond, 1995). Adequate convergent and discriminant validity has also been reported (Crawford \& Henry, 2003).

The fifth measure was the Balanced Inventory of Desirable Responding (BIDR; Paulhus, 1988); a 40-item instrument used to measure self-deceptive positivity, and impression management (deliberate self-presentation to an audience). It is viewed as a measure of defence (i.e., people who score high on self-deceptive positivity tend to defend against negative selfevaluations and seek out inflated positive self-evaluations; Paulhus, 1988). Participants were asked to rate 40 statements on a 7-point scale according to their level of agreement with the statement. Adequate internal consistencies have been reported for self-deceptive positivity and impression management, and test-retest correlations over a five week period were reported as being .69 and .65 for self-deceptive positivity and impression management, respectively (Paulhus, 1988). The BIDR has also demonstrated adequate concurrent validity.

The sixth measure, the Five Facet Mindfulness Questionnaire (FFMQ; Baer, Smith, Hopkins, Krietemeyer, \& Toney, 2006), was based on a factor analytic study of five independently developed mindfulness questionnaires. Participants were required to respond to 39 statements by entering a number from 1 to 5 (from never or very rarely true, to very often or always true) beside the statements. The construct validity of the FFMQ has previously been supported (see Baer et al., 2008), with alpha coefficients ranging from .75 to .91 for the subscales, suggesting adequate to good reliability (Schütze, Rees, Preece, \& Schütze, 2010).

The final measure was the Maslach Burnout Inventory-Educators Survey (MBI-ES; Maslach, Jackson, \& Schwab, 1996), which was specifically adapted to assess burnout levels of individuals who work in school settings. Burnout on the inventory is measured on three dimensions; emotional exhaustion, depersonalisation and personal accomplishment. Participants were required to read 22 statements of job related feelings, decide how often they have felt that way, and enter a number from 0 to 6 (ranging from never to every day) beside each statement. Maslach, Jackson, and Schwab (1996) reported internal consistency estimates of reliability: $0.90,0.79$, and 0.71 , for emotional exhaustion, depersonalisation, and personal accomplishment subscales, respectively.

\subsection{Procedure}

\subsubsection{Preparation and instructions}

All participants completed the IRAP and the following explicit measures in any available empty room in each of their various school settings. It was required that only the participant and the experimenter were present in the room for the duration of the experiment. Participants were not informed as to the aim of the study at this time. If the purpose of the study became clear to any participant during the study the reliability of the results were not thought to be compromised (see McKenna et al., 2007). The IRAP program began with detailed instructions explaining the task, illustrated examples of the screen layout, the stimuli that would be presented, and specific instructions on how to complete the task. On each trial of the IRAP, all of the stimuli (label, target and response options) were presented simultaneously on the computer screen. Participants were required to choose one of the two response options by pressing either the " $\mathrm{d}$ " or " $\mathrm{k}$ " key on the computer keyboard. Choosing the response option that was deemed correct for that block of trials removed all stimuli from the screen. Four hundred ms later the next trial was presented. If the incorrect response option was chosen a red X appeared directly underneath the target stimulus. All stimuli remained on screen until the participant chose the correct response option. Having done so, the screen cleared for the $400 \mathrm{~ms}$ inter-trial interval. If a participant failed to respond within $2000 \mathrm{~ms}$ on any trial, the words "Too Slow" appeared in lower-centre of the screen and remained there until a response (correct or incorrect) was emitted.

The IRAP in its entirety consisted of a minimum of two practice blocks followed by a fixed set of six test blocks. Each block consisted of 24 trials with each of the twelve target stimuli (see Table 1) presented once in the presence of the two label stimuli: "Autistic Spectrum Disorder" and "Normally Developing”. This combination of label and target stimuli yielded four trial-types; Autism-Negative, Autism-Positive, Normally-Developing-Negative, Normally-Developing-Positive (see Fig. 1). The 24 trials were presented quasi-randomly within each block, with the constraint that the same trial-type could not be presented across successive trials. The left-right positioning of the two response options, "Similar" and "Opposite," was also randomised across trials with the constraint that they could not appear in the same positions across three successive trials.

Practice and test blocks were identical in format and differed only in terms of a short instruction presented to participants prior to the first trial in each block. Specifically, before each practice block participants were informed as follows: "This is a practice - errors are expected.", whereas before each test block they were instructed: "This is a test - go fast, making a few errors is ok." Participants were always exposed to the practice blocks prior to the test blocks. Participants were required to achieve a minimum response accuracy criterion of $80 \%$, and a latency criterion of a median of $\leq 2000$-ms, for each of the two practice blocks before they were permitted to continue to the six test blocks. If they failed to achieve these joint criteria a message appeared on screen informing them of this fact. The actual accuracy and latency scores achieved on each block by the participant were also presented, and he or she was invited to complete another two practice blocks. When participants achieved the practice criteria they proceeded to the fixed set of six test blocks. Participants did not need to maintain any performance criteria to progress through the test blocks, but they were instructed to do their best to maintain the criteria they had achieved during the practice phase. 


\section{Autism/Negative Attribute}

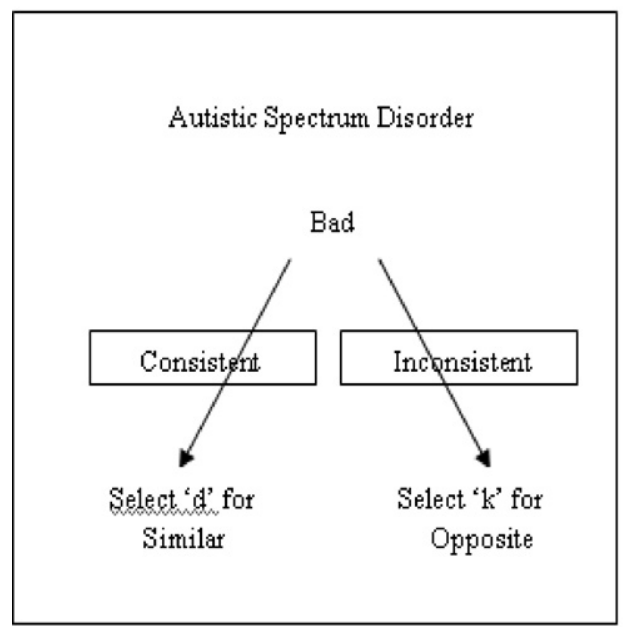

\section{Normally Developing/Negative Attribute}

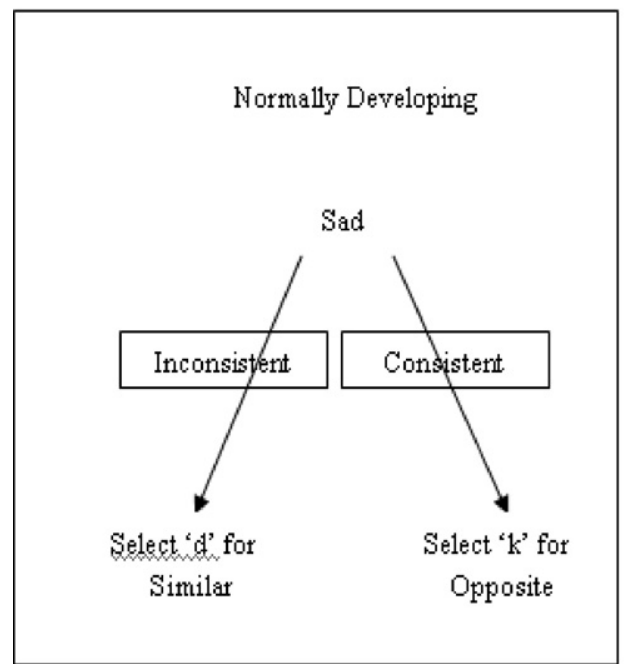

Autism/Positive Attribute

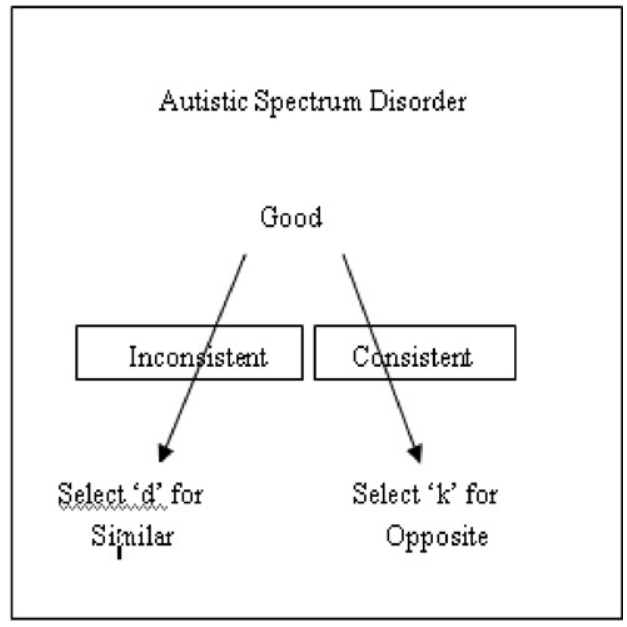

Normally Developing/Positive Attribute

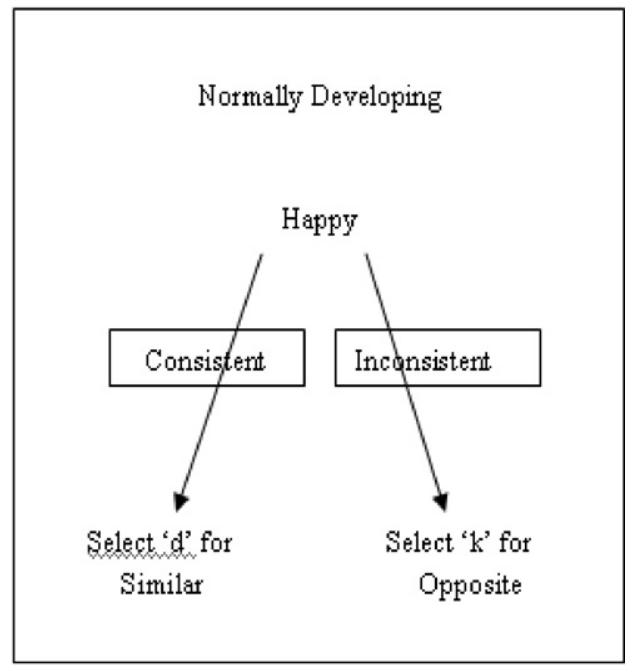

Fig. 1. Examples of the four IRAP trial-types. The labels ("Autistic Spectrum Disorder” and “Normally Developing”), attribute words (e.g., “Good” or “Bad”), and response options ("Similar" or "Opposite") appeared simultaneously on each trial. Arrows with superimposed text boxes serve to indicate which responses were deemed consistent or inconsistent (these boxes and arrows did not appear on screen).

Two opposing patterns of responding were required across the blocks of IRAP trials. In some blocks participants were required to respond in a pattern consistent with autism-negative/normally-developing-positive. That is, responses designated correct on the IRAP were as follows: Autism-Negative-Similar; Autism-Positive-Opposite; Normally-DevelopingPositive-Similar; Normally-Developing-Negative-Opposite. Hereafter, this pattern of responding is referred to as an AutismNegative block. The opposite pattern of responding required the following response pattern: Autism-Negative-Opposite; Autism-Positive-Similar; Normally-Developing-Positive-Opposite; Normally-Developing-Negative-Similar. This latter pattern is referred to as an Autism-Positive block.

The sequencing of the IRAP blocks was always presented in a manner that alternated between Autism-Negative and Autism-Positive. This sequence was also counterbalanced across participants.

\subsubsection{Explicit measures}

Having completed the IRAP, participants completed each of the explicit measures in the following order: FFMQ AAS, BIDR, DASS, Likert Scales for Autism, Likert Scales for Normal, Feeling Thermometer, and MBI-ES. Participants were then thanked and debriefed. 


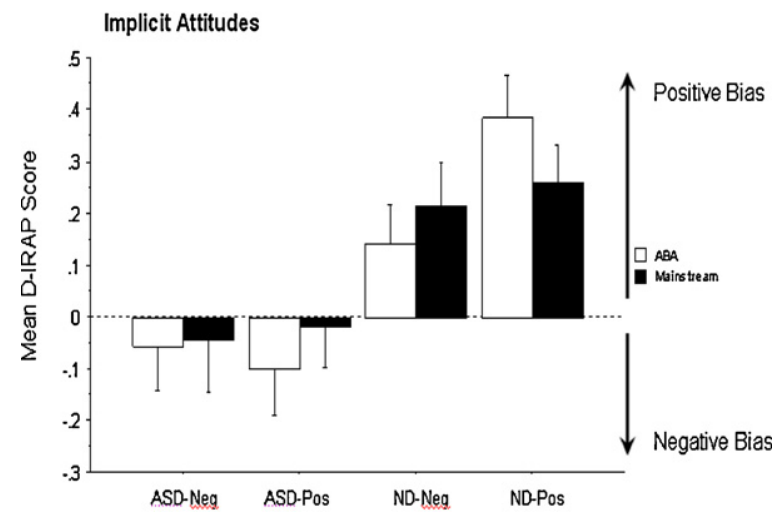

Fig. 2. Mean $D$-IRAP scores with standard error bars for Autism-Negative, Autism-Positive, Normal-Negative and Normal-Positive trial types. A negative $D$ IRAP score indicates a negative bias and a positive $D$-IRAP score indicates a positive bias.

\section{Results}

\subsection{IRAP data}

\subsubsection{Data preparation}

The primary datum was response latency defined as the time in milliseconds that elapsed between the onset of the trial and a correct response emitted by a participant. To control for and minimize contamination due to individual differences such as age, motor skills, and/or cognitive ability, response latency data were transformed into D-IRAP scores (e.g., BarnesHolmes et al., 2010; Cullen \& Barnes-Holmes, 2008). The scores were calculated such that positive values indicated positive bias for both autism and normally developing and negative scores indicated negative bias.

\subsubsection{Mean scores analyses}

The data from all 32 participants were included in the analyses. The four overall mean $D$-IRAP scores are presented in Fig. 2. The D-IRAP effects for the two autism trial-types indicated negative but relatively weak biases for both groups. That is, the two groups showed a slight tendency to respond Similar more quickly than Opposite on Autism-Negative trials, and to respond Opposite more quickly than Similar on Autism-Positive trials. In contrast, both groups of teachers produced positive and relatively strong biases on the two Normally-Developing trial-types. That is, they showed a strong tendency to respond Opposite more quickly than Similar on Normally-Developing-Negative trials, and to respond Similar more quickly than Opposite on Normally-Developing-Positive trials. Finally, the overall $D$-IRAP scores for the two groups were quite similar: ABA, $M=.17$, $S D=.19$; Mainstream, $M=.13, S D=.16$.

The IRAP data were entered into a $2 \times 4$ mixed repeated measures analysis of variance (ANOVA), with teacher-type as the between-participant variable and IRAP trial-type as the within-participant variable. Only the main effect for trialtype proved to be significant, $F(3,90)=10.28, p<.0001, \eta_{p}^{2}=.25$ (remaining $p s>.56$ ). Four independent $t$-tests confirmed that there were no between-group differences for any of the four trial-types (all ps > .26). Fisher's PLSD post hoc tests were used to examine the six differences among the trial-types (data collapsed across the two groups) and these indicated that the comparison between the two autism trial-types was clearly non-significant $(p>.9)$. The two normallydeveloping trial-types approached significance $(p=.08)$. The remaining comparisons however were significant $(p s<003)$. Four one-sample $t$-tests were used to determine if the IRAP trial-type effects (collapsed across the two groups) differed significantly from zero. The effects for the two normally-developing trial-types proved to be significant (Normal-Negative, $t=3.14, d f=31, p<.004$; Normal-Positive, $t=5.75, d f=31, p<.0001)$, but the effects for the two autism trial-types did not $(p s>.3)$.

\subsection{Explicit measures}

\subsubsection{Attitude measures}

Participants completed three explicit attitude measures, two of which targeted their attitudes to normally-developing children and those with a diagnosis of autism; the Likert scales and the feeling thermometer. The AAS targeted only autism and thus produced one overall score. The means, standard deviations and inferential statistics ${ }^{1}$ for each of these explicit measures for the ABA and mainstream teachers are presented in Table 2 (upper half). The ABA tutors scored significantly

\footnotetext{
${ }^{1}$ The results for the Likert scales and feeling thermometers were also entered into $2 x 2$ mixed repeated measures ANOVAs, but neither yielded significant interaction effects $(p s>.3)$.
} 
Table 2

ABA tutors and mainstream teachers explicit measures table of means, standard deviations and ANOVAs.

\begin{tabular}{|c|c|c|c|c|c|c|c|}
\hline \multirow[t]{2}{*}{ Explicit measures } & \multicolumn{2}{|c|}{ ABA tutors } & \multicolumn{2}{|c|}{ Mainstream teachers } & \multirow[t]{2}{*}{$F$} & \multirow[t]{2}{*}{$p$} & \multirow[t]{2}{*}{$\eta$} \\
\hline & $M$ & $S D$ & $M$ & $S D$ & & & \\
\hline \multicolumn{8}{|l|}{ Attitude measures } \\
\hline Likert autism & .24 & 1.21 & .09 & .99 & .14 & .71 & .005 \\
\hline Likert normal & 1.82 & .50 & 1.16 & .91 & 6.68 & .015 & .18 \\
\hline FT autism & 77.50 & 16.53 & 58.75 & 21.56 & 7.62 & .010 & .20 \\
\hline FT normal & 88.12 & 9.81 & 75.62 & 14.13 & 8.45 & .007 & .22 \\
\hline AAS & 3.2 & .26 & 3.05 & .57 & 1.08 & .31 & 0.03 \\
\hline \multicolumn{8}{|l|}{ Non-attitude measures } \\
\hline DASS depression & 3.62 & 4.54 & 5.19 & 5.91 & .70 & .41 & 0.02 \\
\hline DASS anxiety & 2.25 & 2.49 & 4.43 & 3.71 & 3.84 & .06 & 0.11 \\
\hline DASS stress & 8.81 & 6.67 & 11.87 & 7.93 & 1.40 & .25 & 0.04 \\
\hline Mindfulness & 3.21 & .26 & 3.34 & .34 & 1.48 & .23 & .05 \\
\hline MBI-ES emotional exhaustion & 2.19 & 1.24 & 1.78 & 1.12 & .97 & .33 & .03 \\
\hline MBI-ES depersonalisation & .62 & .73 & .92 & .94 & .97 & .33 & .03 \\
\hline MBI-ES personal accomplishment & 4.39 & .71 & 5.25 & .84 & 9.86 & .004 & .25 \\
\hline BIDR & 3.35 & .56 & 4.16 & .64 & 14.70 & .0006 & .33 \\
\hline
\end{tabular}

higher than the mainstream teachers when rating normally developing children on the Likert scale; this pattern was also recorded for both feeling thermometers; the AAS did not yield a significant difference. In effect, the ABA tutors tended to rate both types of children more positively than the mainstream teachers.

\subsubsection{Non-attitude measures}

Participants completed four different measures targeting general psychopathology (DASS), professional burnout (MBI-ES), mindfulness (FFMQ) and social desirability (BIDR). The means, standard deviations and inferential statistics are presented in the lower half of Table 2. The two groups did not differ significantly on the DASS or any of its three subscales. The mainstream teachers were inclined to report somewhat higher levels of anxiety relative to the ABA tutors, albeit to a level that just failed to reach significance $(p=.06)$. One significant difference was recorded for the MBI-ES, indicating that the mainstream teachers reported higher levels of personal accomplishment relative to the ABA group. Finally, the mainstream teachers also reported significantly higher levels of desirable responding relative to the ABA tutors.

\subsection{Implicit-explicit correlations}

Preliminary analyses indicated that the pattern of explicit-implicit correlations between the two groups differed quite dramatically. The results of separate correlational analyses for each group are thus presented.

\subsubsection{ABA tutors}

Each of the five D-IRAP scores was correlated with each of the 15 scores from the explicit measures. Out of the 75 correlations for the ABA tutors, 13 were significant. These are presented in Table 3. Interestingly, the Autism-Negative

Table 3

ABA tutors explicit-implicit correlation table.

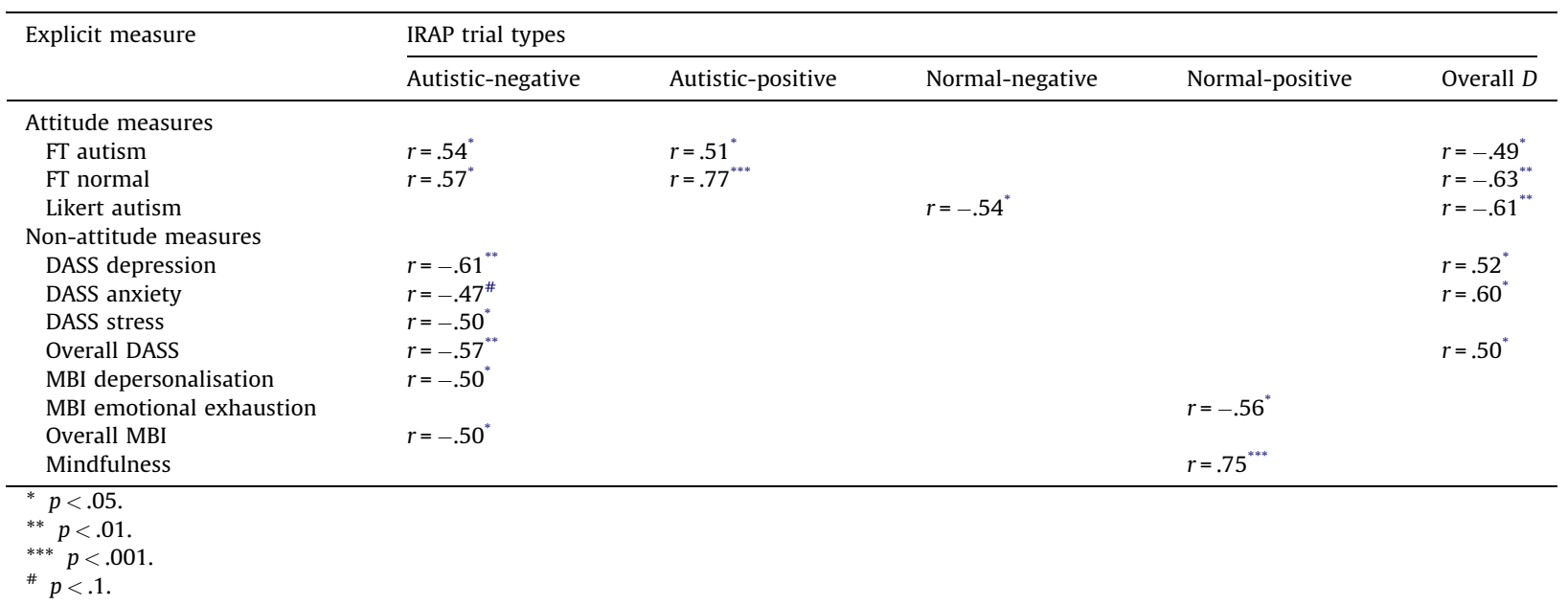


trial-type correlated significantly with three of the DASS measures (overall DASS, Stress and Depression subscales) and correlated at a marginally significant level with the fourth, the anxiety subscale $(r=-.47, p=.06)$. This trial-type also correlated with the two feeling thermometers, the MBI depersonalisation sub-scale and the overall MBI scores. In effect, increased negative implicit bias towards children with autism appeared to predict increased symptoms of psychopathology, depersonalisation, overall burnout, and lower ratings of both types of children on the feeling thermometers. Increased scores on the Autism-Positive IRAP trial-type correlated with increased positivity towards both types of children on the two feeling thermometers. Higher scores on the Normal-Negative trial-type correlated with lower ratings of autism on the Likert scale. Higher scores on the Normal-Positive trial-type correlated with increased levels of mindfulness (FFFMQ) and lower levels of emotional exhaustion (MBI). Finally, the overall $D$-IRAP score correlated with six of the explicit measures with three of these predicting the DASS scores.

\subsubsection{Mainstream teachers}

Out of the 75 correlations for the mainstream teachers only one was clearly significant. Specifically, lower negativity towards autism on the Autism-Negative trial-type correlated with increased socially desirable responding on the BIDR $(r=.68, p=.003)$. The correlation for this trial-type with the Likert-normal scores was marginally significant $(r=-.49$, $p=.052$ ). The correlation between the Normal-Positive trial-type and the feeling thermometer for normally developing approached significance $(r=-.43, p=.09)$.

\subsection{Regression analyses}

As noted above, a range of significant correlations were obtained between the $D$-IRAP scores and the explicit measures for the ABA tutors. Although it was not the focus of the current study, correlational analyses among the explicit measures were also conducted. These yielded significant correlations between the feeling thermometers and some of the DASS scores, again for the ABA group (i.e., DASS Depression with feeling thermometer for normally developing children, $r=-.52, p=.03$, and for children with autism, $r=-.52, p=.04$; DASS Stress with feeling thermometer for normally developing children, $r=-.51$, $p=.04$; Overall DASS with feeling thermometer for normally developing children, $r=-.55, p=.03$ ). Given that two of the explicit measures of the ABA tutors' reactions to children (i.e., the feeling thermometers) predicted psychopathology on the DASS, it was deemed important to determine if the D-IRAP scores that also predicted DASS responses accounted for additional variance above and beyond these explicit measures. Four separate two-step multiple regression analyses were thus conducted.

In the first step of the first regression analysis responses on the feeling thermometer for children with autism were entered as the independent variable and the DASS depression scores were entered as the dependent variable. The result proved to be significant, $b=-.14, t=-2.27, p=.03$, accounting for $27 \%$ of the variance. When the $D$-IRAP scores from the Autism-Negative trial-type were entered into the second step of the model, the result approached significance, $b=-6.12$, $t=-1.86, p=.08$, accounting for $42 \%$ of the variance $\left(R^{2}\right.$ change $\left.=15 \%\right)$. The same analytic strategy was then adopted for the feeling thermometer for normally developing children, yielding a significant effect for the first step, $b=-.24, t=-2.3$, $p=.03$, accounting for $28 \%$ of the variance. In the second step, the Autism-Negative trial-type again approached significance, $b=-6.05, t=-1.78, p=.09$, accounting for $42 \%$ of the variance $\left(R^{2}\right.$ change $\left.=14 \%\right)$.

For the third multiple regression analysis, the feeling thermometer for normally-developing children was used as the independent variable and scores on the DASS Stress scale were entered as the dependent variable, and this proved to be significant, $b=-.35, t=-2.25, p=.04$, accounting for $27 \%$ of the variance. When the Autism-Negative trial-type was entered into the second step of the model it had little impact on the variance accounted for, $b=-5.85, t=-1.09, p=.29$, accounting for $32 \%$ of the variance $\left(R^{2}\right.$ change $=5 \%$ ).

The final multiple regression analysis entered the feeling thermometer for normally developing children as the independent variable and the overall DASS scores as the dependent variable, and this yielded a significant effect, $b=-.71, t=-2.45, p=.03$, accounting for $30 \%$ of the variance. When the Overall $D$-IRAP scores were entered into the second step of the model, it had a limited impact, $b=-17.02, t=.89, p=.39$, accounting for $34 \%$ of the variance $\left(R^{2}\right.$ change $=4 \%$ ).

Overall therefore, the D-IRAP scores from the Autism-Negative trial-type appeared to provide incremental validity in terms of predicting Depression scores on the DASS, but added little in predicting Stress scores. In addition, the overall $D$-IRAP scores did not increase predictive validity when using the feeling thermometers for normally developing children to predict the overall DASS measure.

\subsection{Summary and conclusion}

In summary, there was no significance difference in implicit attitudes between mainstream and ABA tutors. Both groups showed implicit positive biases towards normally developing children but weak negative biases towards children with autism, with this latter effect also reflected in the explicit measures. For the ABA tutors, their implicit attitudes; particularly negative bias towards autism, predicted professional burnout (e.g., Overall MBI), psychopathology (e.g., Depression), and some explicit measures (e.g., feeling thermometers). For the mainstream teachers however, the IRAP only predicted socially desirable responding. 


\section{Discussion}

The current study aimed to further the preliminary findings reported by Barnes-Holmes et al. (2006) by exploring implicit attitudes towards autism among ABA tutors and mainstream teachers, and endeavouring to determine the extent to which negative implicit attitudes predicted burnout and psychopathology. Results indicated that both groups of participants produced implicit biases on the IRAP that indicated greater positivity towards normally developing children relative to children with autism. These findings are consistent with previous findings by Barnes-Holmes et al. in which autism professionals and those with no experience of autism also produced increasingly negative biases towards individuals with autism when compared to normally developing children. Furthermore, the lack of significant differences in responding between the ABA tutors and the mainstream teachers was entirely in line with the findings of Barnes-Holmes et al. in which they also reported no significant differences in responding between groups.

In the Barnes-Holmes et al. (2006) study they reported the classic separation between the implicit and explicit measure results, specifically on the AAS. The current results did indicate some explicit-implicit divergence in that the ABA tutors rated normally developing children higher than the mainstream teachers on the Likert scale and both types of children higher on the feeling thermometers, which contrasted with the lack of differences between the groups responding on the IRAP. However, in contrast to the study by Barnes-Holmes et al. there were no significant differences reported between the two groups' ratings on the AAS. This may indicate that the ABA tutors in the current study were more willing to disclose some negativity on the explicit measure. Why might this be the case?

Notably, the professionals in the current study worked with children with diagnoses of severe autism and therefore encountered high frequency and intense behaviours daily. Recent research by Dowjotas (2009) on students' attitudes towards children with autism found that attitudes differed based on severity, such that students were most positive toward children with mild autistic symptoms and more negative toward children with more severe autistic symptoms. These results for severity were found across all measures used to assess participants' attitudes, and also seem consistent with prior research (e.g., Socall \& Holtgraves, 1992; Wang, Thomas, Chan, \& Cheing, 2003). The severity of the autism diagnoses with which the professionals were working in the Barnes-Holmes et al. (2006) study were unspecified by the participants, and therefore it is unclear whether severity played a role in the discord between the disclosure of attitudes in the current study compared to the research by Barnes-Holmes et al. Furthermore, it is worth noting that the mainstream group reported significantly greater levels of socially desirable responding relative to the ABA tutors. In the context of the current study this suggests that the ABA group appeared less motivated by social desirability concerns and perhaps therefore reported more negative attitudes towards autism than the tutors in the original study.

The implicit-explicit correlations for the ABA tutors indicated that increased scores on the Autism-Positive IRAP trial-type predicted increased positivity towards both groups of children on the two feeling thermometers. This finding was unsurprising, and largely in line with the idea that a participant who is implicitly positive about autism will have no motivation to mask responding on explicit attitude measures. It was also revealed however, that increased negativity on the Autism-Negative trial type correlated with lower ratings of both types of children on the feeling thermometers. This indicated that the more negative the participant was regarding children with autism the lower they rated children in general on the explicit measure. These findings could be seen as contradictory to previous research, whereby individuals typically tend to be motivated to mask negative socially sensitive attitudes, thus producing a divergence between implicit and explicit measures (Barnes-Holmes et al., 2010). However, as noted above more severe diagnoses may produce more explicitly negative attitudes (Dowjotas, 2009; Wang et al., 2003). Future research exploring this issue in greater detail would be valuable.

Another issue raised by the implicit-explicit correlations was as follows. When examining the correlations for the ABA tutors between the IRAP and the psychopathology measures, it became clear that one trial type in particular correlated highly with measures of psychopathology, namely the Autism-Negative trial type. Thus, ABA tutors who more readily selected "Similar" than "Opposite" in the presence of autism and a negative attribute had higher rates of depression, anxiety and stress as rated by the DASS, higher depersonalisation and overall burnout as rated by the MBI-ES, and lower ratings of both groups of children on the feeling thermometers. Interestingly, no correlations were observed for the Autism-Positive trial-type (i.e., lower positivity did not predict higher levels of psychopathology). These findings thus appear to highlight the specificity of the IRAP as a measure of implicit response biases. But why was this trial-type specific correlation observed?

Given that the ABA group interacted on a daily basis with children with autism it seems likely that regular thoughts and feelings, if only fleeting, that the client group are negative in some way may serve to increase feelings of guilt and inadequacy in their professional roles. As described by Pruett and Chan (2006), when professional carers feel pressure to outwardly express attitudes in line with what is thought to be socially appropriate while attempting to overcome negative biases and assumptions, this can lead to symptoms of burnout and other psychological problems (see Maslach, Jackson, \& Leiter 1996). It seems likely that such effects would be most pronounced for thoughts and feelings that are deemed to be negative, as opposed to thoughts and feelings that fail to confirm some level of positivity. In other words, not feeling particularly positive about working with very challenging clients would likely create far less psychological stress than feeling particularly negative in this regard. For example, it may well be acceptable to say "Some days I don't think the kids are particularly good", but it would not be as appropriate to say "Some days I think the kids are absolutely awful." Insofar as the Autism-Negative IRAP trial targeted the latter thought or feeling, and the Autism-Positive trial-type the former, then it makes sense that the correlations with psychopathology were relatively specific. In any case, similar to the conclusions of Barnes-Holmes et al. 
(2006), these findings further illustrate that there is a need for professional training programmes aimed at dealing with burnout and symptoms that foster burnout, such as depression, stress and depersonalisation, among those working with individuals with autism.

For the mainstream participants, there was only one significant correlation between the implicit and explicit measures; lower negativity on the Autism-Negative trial type predicted increased desirable responding on the BIDR. This outcome was somewhat unexpected in that previous research typically has not found correlations between implicit measures and social desirability. Critically however, the effect was only found for the mainstream group, who it could be argued would have relatively weak or even non-existent attitudes towards autism (because the mainstream teachers in the study had little or no contact with such children). Interestingly, Schwartz (2007) noted that the literature on automatic evaluations suggests that people continuously evaluate all stimuli that they encounter (see Bargh, 1997), including stimuli for which no previous attitude has been formed (Duckworth, Bargh, Garcia, \& Chaiken, 2002), and these evaluations are highly sensitive to both the current context and the actor's goals (e.g., Ferguson \& Bargh, 2004). Insofar as it is more socially acceptable to have a positive rather than a negative attitude towards disability in general, the IRAP performances for the mainstream group may thus have been influenced by individual concerns over such political correctness. Of course, this interpretation does remain somewhat speculative and in need of systematic empirical analysis.

The two feeling thermometers predicted participant responses on the DASS, and thus regression analyses were conducted to determine if the D-IRAP scores that also predicted DASS responses accounted for additional variance beyond the feeling thermometers. The D-IRAP scores from the Autism-Negative trial-type appeared to provide incremental validity in terms of predicting depression scores on the DASS, but added little in predicting stress. The current findings thus suggest that measures such as the feeling thermometers that assess feelings towards a client group may have valid utility when researching current psychological states of carers or practitioners. However, the ability of the D-IRAP score for the AutismNegative trial-type to add to the predictive validity of the feeling thermometers for the DASS depression measure further illustrates the utility of the implicit measure. The current findings thus indicate that in cases where the relationships between attitudes and psychopathology or burnout are being targeted, it may be useful to use both explicit and implicit measures, rather than relying solely on one or the other. This implication needs to be further explored in that it may be the case that explicit and implicit measure of attitude can work to complement one another; however the specificity of target attitude areas and research environments in which this may be the case remains to be explored.

There were some further issues in the current study which additional research could address. Firstly the sample size of the two groups was relatively small. Due to time limitations when conducting the study it was impossible to recruit larger sample sizes. A replication encompassing a larger sample size would ensure more generalizable results. A further limitation in this regard was that the participants in the study were primarily female. It may be useful for future research to examine both genders and empirically examine if differences exist between the genders. Nonetheless however, the current study highlights an important need for further research on the psychological and behavioural impact that people's attitudes can have; and also further illustrates the utility of the IRAP as an implicit, thus unbiased, attitude measure. The current study was quite exploratory in nature, thus a plethora of explicit measures were administered in order to thoroughly determine which areas of explicit attitude and psychopathology measures may be correlated with implicit attitudes. The resulting correlational analyses thus appear very in depth for such a sample size. It was however deemed necessary that all possible correlations or lack thereof between each particular trial type, each explicit measure, and each group, were explored. The resulting data, although somewhat overwhelming, was extremely thorough in depicting areas in which further research is necessary (i.e., the autism-negative trial type correlation with measures of burnout and psychopathology). Perhaps future research would limit the amount of explicit measures insofar as it would allow a more in-depth analysis of the implicit measure and its correlation with self reported psychopathology specifically.

Overall however, the IRAP appears to have potential as a measure of implicit attitudes towards client populations among professional carers and educators. In particular, the IRAP appears to highlight a link between negative implicit attitudes to a client group and higher levels of psychopathology, including professional burnout, experienced by carers/educators within the workplace.

\section{References}

Akrami, N., Ekehammar, B., Claesson, M., \& Sonnander, K. (2006). Classical and modern prejudice: Attitudes toward people with intellectual disabilities. Research in Developmental Disabilities, 27, 605-617.

Amodio, D. M., Harmon-Jones, E., \& Devine, P. G. (2003). Individual differences in the activation and control of affective race bias as assessed by startle eyeblink responses and self report. Journal of Personality and Social Psychology, 84, 738-753.

Baer, R. A., Smith, G. T., Hopkins, J., Krietemeyer, J., \& Toney, L. (2006). Using self-report assessment methods to explore facets of mindfulness. Assessment, 13, 2745.

Baer, R. A., Smith, G. T., Lykins, E., Button, D., Krietemeyer, J., Sauer, S., et al. (2008). Construct validity of the five facet mindfulness questionnaire in meditating and nonmeditating samples. Assessment, 15, 329-342.

Bargh, J. A. (1997). The automaticity of everyday life. In Wyer, R. S. (Ed.). Jr.The automaticity of everyday life: Advances in social cognition (Vol. 10, pp. 1-61).Mahwah, NJ: Erlbaum.

Barnes-Holmes, D., Barnes-Holmes, Y., Power, P., Hayden, E., Milne, R., \& Stewart, I. (2006). Do you really know what you believe? Developing the Implicit Relational Assessment Procedure (IRAP) as a direct measure of implicit beliefs. The Irish Psychologist, 32, 169-177.

Barnes-Holmes, D., Barnes-Holmes, Y., Stewart, I., \& Boles, S. (2010). A sketch of the Implicit Relational Assessment Procedure (IRAP) and the Relational Elaboration and Coherence (REC) model. The Psychological Record, 60, 527-542.

Bishop, S. R., Lau, M., Shapiro, S., Carlson, L., Anderson, N. D., Carmody, J., et al. (2004). Mindfulness: A proposed operational definition. Clinical Psychology: Science and Practice, 11, 230-241. http://dx.doi.org/10.1093/clipsy/bph077. 
Cohen, L., Manion, L., \& Morrison, K. (2000). Research methods in education (5th edition). London: Routledge Falmer.

Crawford, J. R., \& Henry, J. D. (2003). The Depression Anxiety Stress Scale (DASS): Normative data and latent structure in a large non-clinical sample. British Journal of Clinical Psychology, 42, 111-131.

Cullen, C., \& Barnes-Holmes, D. (2008). Implicit pride and prejudice: A heterosexual phenomenon? In T. G. Morrison \& M. A. Morrison (Eds.), Modern prejudice (pp. 195-223). New York: Nova Science.

De Houwer, J., Crombez, G., Baeyens, F., \& Hermans, D. (2001). On the generality of the affective Simon effect. Cognition and Emotion, 15 , 189-206.

Dowjotas, J. M. (2009). Why can't we be friends?: The effects of disclosure and severity on typical students' attitudes toward peers with autism spectrum disorders. Honors Projects. Paper 126. Retrieved from http://digitalcommons.iwu.edu/psych_honproj/126 on 15.04.10.

Duckworth, K. L., Bargh, J. A., Garcia, M., \& Chaiken, S. (2002). The automatic evaluation of novel stimuli. Psychological Science, $13,513-519$.

Fazio, R. H., Sanbonmatsu, D. M., Powell, M. C., \& Kardes, F. R. (1986). On the automatic activation of attitudes. Journal of Personality and Social Psychology, 50, 229238.

Ferguson, M. J., \& Bargh, J. A. (2004). Liking is for doing: Effects of goal pursuit on automatic evaluation. Journal of Personality and Social Psychology, 88, 557-572.

Fiedler, K., \& Bluemke, M. (2005). Faking the IAT: Aided and unaided response control on the Implicit Association Test. Basic and Applied Social Psychology, 27, 307316.

Friese, M., Hofmann, W., \& Wanke, M. (2008). When impulses take over: Moderated predictive validity of explicit and implicit attitude measures in predicting food choice and consumption behaviour. Social Psychology, 47, 397-419.

Greenwald, A. G., \& Banaji, M. R. (1995). Implicit social cognition: Attitudes, self-esteem, and stereotypes. Psychological Review, 102, 4-27.

Greenwald, A. G., McGhee, D. E., \& Schwartz, J. L. K. (1998). Measuring individual differences in implicit cognition: The Implicit Association Test. Journal of Personality and Social Psychology, 74, 1464-1480.

Hayes, S. C., Bisset, R., Roget, N., Padilla, M., Kohlenberg, B. S., Fischer, G., et al. (2004). The impact of acceptance and commitment training and multi-cultural training on the stigmatizing attitudes and professional burnout of substance abuse counsellors. Behavior Therapy, 35, 821-835.

Hakanen, J. J., Bakker, A. B., \& Schaufeli, W. B. (2006). Burnout and work engagement among teachers. Journal of School Psychology, $43,495-513$.

Jackson, S. E., \& Maslach, C. (1982). After-effects of job-related stress: Families as victims. Journal of Occupational Behaviour, 3, $63-77$.

Kaplan, S. P. (1982). Rehabilitation counselors' attitudes toward their clients. Journal of Rehabilitation, 48, 28-30.

Kim, D. Y. (2003). Voluntary controllability of the Implicit Association Test (IAT). Social Psychology Quarterly, 66, 83-96.

Kolar, C., \& Dickson, S. V. (2002). Preservice general educators' perceptions of structured reflective logs as viable learning tools in a university course on exclusionary practices. Teacher Education and Special Education, 25, 395-406.

Leyser, Y., Kapperman, G., \& Keller, R. (1994). Teacher attitudes toward mainstreaming: A cross-cultural study in six nations. European Journal of Special Needs Education, 9, 1-15.

Lovibond, S. H., \& Lovibond, P. F. (1995). Manual for the Depression Anxiety Stress Scales (2nd edition). Sydney: Psychology Foundation.

Lowenstein, L. (1991). Teacher stress leading to burnout-Its prevention and cure. Education Today, 41, 12-16.

Maslach, C. (1982). Burnout: The cost of caring. Englewood Cliffs, NJ: Prentice Hall.

Maslach, C., \& Jackson, S. E. (1985). The role of sex and family variables in burnout. Sex Roles, 12, 837-851.

Maslach, C., Jackson, S. E., \& Leiter, M. P. (1996). Maslach burnout inventory manual (3rd edition). Palo Alto, CA: Consulting Psychologists Press.

Maslach, C., Jackson, S. E., \& Schwab, R. L. (1996). Maslach Burnout Inventory-Educators Survey (MBI-ES). In C. Maslach, S. E. Jackson, \& M. P. Leiter (Eds.), Maslach burnout inventory manual (3rd edition). Palo Alto, CA: Consulting Psychologists Press.

Maslach, C., \& Pines, A. (1977). The burnout syndrome in the day care setting. Child Care Quarterly, 6, 100-113.

McKenna, I. M., Barnes-Holmes, D., Barnes-Holmes, Y., \& Stewart, I. (2007). Testing the fake-ability of the implicit relational assessment procedure (IRAP): The first study. International Journal of Psychology and Psychological Therapy, 7, 123-138.

Paulhus, D. L. (1988). Assessing self-deception and impression management in self-reports: The balanced inventory of socially desirable responding. Unpublished manual. University of British Columbia, Vancouver, Canada.

Petty, R. E., Haugtvedt, C., \& Smith, S. M. (1995). Elaboration as a determinant of attitude strength: Creating attitudes that are persistent, resistant, and predictive of behavior. In R. E. Petty \& J. A. Krosnick (Eds.), Attitude strength: Antecedents and consequences (pp. 93-130). Mahwah, NJ: Lawrence Erlbaum.

Pittock, F., \& Potts, M. (1988). Neighbourhood attitudes to people with a mental handicap: A comparative study. British Journal of Mental Subnormality, 34, 35-46.

Praisner, C. L. (2003). Attitudes of elementary school principles toward the inclusion of students with disabilities. Exceptional Children, $69,135-145$.

Pruett, S. R., \& Chan, F. (2006). The development and psychometric validation of the disability attitude implicit association test. Rehabilitation Psychology, 51, 202213.

Reeve, D. (2000). Oppression within the counselling room. Disability Er Society, 15, 669-682.

Schütze, R., Rees, C., Preece, M., \& Schütze, M. (2010). Low mindfulness predicts pain catastrophizing in a fear-avoidance model of chronic pain. Pain, $148,120-127$.

Schwartz, N. (2007). Attitude construction. Evaluation in context. Social Cognition, 25(5), 638-656.

Shiffrin, R. M., \& Schneider, W. (1977). Controlled and automatic human information processing: II. Perceptual learning, automatic attending, and a general theory. Psychological Review, 84, 127-190.

Silver, B. D., Abramson, P. R., \& Anderson, B. A. (1986). The presence of others and over reporting of voting in American national elections. Public Opinion Quarterly, $50,228-239$.

Socall, D. W., \& Holtgraves, T. (1992). Attitudes toward the mentally ill: The effects of label and beliefs. The Sociological Quarterly, 33, $435-445$.

Thomas, A., Doyle, A., \& Vaughn, D. (2007). Implementation of a computer based Implicit Association Test as a measure of attitudes toward individuals with disabilities. Journal of Rehabilitation, 73, 3-14.

Vaughan, E. D., Thomas, A., \& Doyle, A. L. (2011). The Multiple Disability Implicit Association Test: Psychometric analysis of a multiple administration IAT measure. Rehabilitation Counselling Bulletin, 54, 223-235.

Wang, M. H., Thomas, K. R., Chan, F., \& Cheing, G. (2003). A conjoint analysis of factors influencing American and Taiwanese college students' preferences for people with disabilities. Rehabilitation Psychology, 48, 195-201.

White, M. J., Jackson, V., \& Gordon, P. (2006). Implicit and explicit attitudes toward athletes with disabilities. Journal of Rehabilitationhttp://findarticles.com/p/ articles/mi_m0825/is_3_72/ai_n16864828/pg_9/?tag=content;col1.

Wilson, G. L. (2005). Attitude change through service learning. Academic Exchange Quarterly Retrieved from http://www.thefreelibrary.com/Attitude+change+through+service+learning-a0132867313 on 3.04.09..

Wittenbrink, B., \& Schwarz, N. (2007). Implicit measures of attitudes. New York: Guilford Press.

Wong, D. W., Chan, F., Cardoso, E., Lam, C. S., \& Miller, S. (2004). Rehabilitation counselling students' attitudes toward people with disabilities in three social contexts: A conjoint analysis. Rehabilitation Counselling Bulletin, 47, 194-204.

Wright, B. A. (1988). Attitudes and the fundamental negative bias: Conditions and corrections. In H. E. Yuker (Ed.), Attitudes toward persons with disabilities (pp. 321). New York: Springer. 